dark osmiophilic bodies, and loss of detail in unidentified round grey bodies enclosed in membranes.

Drs. L. Bieder and R. D. Wigley reported A Study of Anaemia in Rheumatoid Arthritis : vitamin B.12 absorption using the Schilling test was studied in sixty arthritics and in controls of similar age distribution. There was no significant difference in patients under 65 years, but the majority of older arthritics showed a level below the lower limit of normal at 15 per cent. Three had frank pernicious anaemia and another four had uptakes below 6 per cent. with improved uptake after intrinsic factor, except in one case with a malabsorption syndrome. Serum vitamin B.12 levels were difficult to interpret as some patients had been given vitamin B.12 elsewhere for indefinite reasons. The effect of various drug treatments on B.12 absorption is being studied.

Dr. B. Rose read a paper on a study with DR. I. A. M. Prior on Uric Acid and the Public Health: surveys of gout and serum uric acid levels in Europeans and Polynesians in differing environments had confirmed the importance of the genetic contribution. The prevalence in New Zealand Maori males was 10.4 per cent.; Rarotongan males $2 \cdot 4$ per cent.; European New Zealanders 2 per cent.; Pukapukan males $5 \cdot 3$ per cent. The lastnamed live on an isolated coral atoll close to the equator. The Maori data showed the association of obesity, diabetes, hypertension, and degenerative cardiovascular disorders. These abnormalities were found more frequently in the gouty males than in the others, but hypertension, obesity, and degenerative cardiovascular disorders were virtually absent in Pukapukan males, with a prevalence of diabetes of $1 \cdot 6$ per cent. compared with $9 \cdot 2$ per cent. in Maoris - a difference attributed to environment.

The attack rate of gout per 100 at risk is shown below in relation to serum uric acid level.

\begin{tabular}{|c|c|c|c|}
\hline & \multirow{2}{*}{ Series } & \multicolumn{2}{|c|}{ Serum Uric Acid } \\
\hline & & $6 \mathrm{mg}$. or less & $8 \mathrm{mg}$. \\
\hline $\begin{array}{l}\text { Cases of } \\
\text { Gout } \\
\text { per } 100 \\
\text { at Risk }\end{array}$ & $\begin{array}{l}\text { N.Z. Maori } \\
\text { Rarotonga } \\
\text { Pukapuka } \\
\text { N.Z. Europeans }\end{array}$ & $\begin{array}{l}2 \cdot 8 \\
\text { Nil } \\
\text { Nil } \\
\text { Nil }\end{array}$ & $\begin{array}{r}25 \cdot 5 \\
5 \cdot 5 \\
23 \\
23\end{array}$ \\
\hline
\end{tabular}

With the exception of Rarotongan males, the attack rate corresponded closely to a serum level of $8 \mathrm{mg}$. Popula- 3 tion surveys of uric acid level were recommended for the detection and early treatment of gout.

Drs. D. B. Myers, T. C. Highton, and M. Garrett $\overrightarrow{\overrightarrow{5}}$ described The Inter-actions of Serotonin and Collagen: the in vitro effect of serotonin on tendon and the physicochemical investigation of the interaction between serotonin $\frac{\bar{\sigma}}{\bar{N}}$ and purified collagen are being studied. Serotonin does $\frac{\mathscr{S}}{\vec{T}}$ not bind appreciably to collagen. It seems to break inter- $\stackrel{\AA}{\Omega}$ and intra-molecular cross links, possibly involving hexoses, allowing increased solubility and decreased $s$ swelling in saline and diluted acetic acid. The fibro- $\overrightarrow{0}$ genesis and structural hierarchy of collagen fibres and the $\overrightarrow{\vec{t}}$ metabolic pathways of serotonin were discussed.

Dr. J. D. WiLson and Miss H. A. Simmonds described gो The Effects of Allopurinol in Gout Patients with Uraemia: of 44 patients with gout and a blood urea over $50 \mathrm{mg}$., i ten were treated with Allopurinol. Where the drug could ir be given in adequate dosage a drop in serum uric acid level is to less than $6.5 \mathrm{mg}$. resulted. Uric acid excretion fell $\infty$ slowly over some months to as low as $3 \mathrm{mg}$. per $100 \mathrm{ml}$. 윽 This was accounted for by a compensatory rise in the excretion of xanthine and hypoxanthine. Daily colchi- $\omega$ cine and a small dose of indomethacin were used to control $\mathbb{D}$ gout symptoms. No improvement in renal function was $\underset{\mathbb{D}}{\vec{D}}$ noted. One patient developed diarrhoea, and a mild 3 inguinal rash occurred in two patients. The drug appeared effective in controlling blood uric acid in uraemia and limited the formation of tophi, but its long-term effegt on the nephropathy must await further studies.

DRS. R. HowES and I. ISDALE reported on the Intris articular Use of Thiotepa in Rheumatoid Arthritis: con trary to expectation, this drug was found to be of value in rheumatoid arthritic joints with laxity of ligaments.

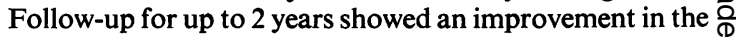
function and stability of the joints. Repeated injections $\varrho$ were more effective than single injections. There was a $\overrightarrow{\overline{0}}$ decrease in the pain, swelling, and recurrence of effusions. 3 A double-blind study is in progress.

Dr. Christopher Gresson read a paper on "Skin Manifestations of Rheumatic Disease".

DR. R. Howes opened a discussion on The Value of $\frac{a}{\varrho}$ Electronmyography.

\title{
EUROPEAN LEAGUE AGAINST RHEUMATISM
} Lisbon, October 8-14, 1967

The tentative programme of the meeting to be held in Lisbon in 1967 consists of three Symposia, one on Sero-negative Polyarthritis, one on the Aetiology and Prevention of Chronic Rheumatism including cartilage degeneration and repair, and the third on Rheumatic Ankylosing Spondylopathies (excluding classical ankylosing spondylitis).

There will also be free papers.

The papers given at the Symposia will be by invi- tation, though anyone may submit a free paper; the latter must be submitted through the appropriate National League.

The main meeting will be held in Lisbon, though $\tilde{O}$ some congressionalists may stay at Estoril, the local $\underset{\omega}{N}$ coast resort.

Further information will be published when available.

G. D. Kersley, President, European League Against Rheumatism. 